\title{
Global Lung Initiative equations for pulmonary hypertension screening in systemic sclerosis
}

\author{
To the Editor:
}

Systemic sclerosis (SSc) is a connective tissue disease characterised by widespread vasculopathy and excessive fibrosis in multiple organs, including the lungs [1]. The most frequent pulmonary involvement in SSc is interstitial lung disease (ILD), but the most harmful is pulmonary hypertension (PH), a complication found in about $10 \%$ of SSc patients [2]. In patients with SSc, early diagnosis of and prompt therapy for PH (either isolated or associated with ILD) are beneficial from a prognostic standpoint and recommendations for active screening of $\mathrm{PH}$ in SSc have therefore been established [3].

Pulmonary function tests (PFTs) are one of the key tools used for PH screening in SSc patients [4] and transfer factor of the lung for carbon monoxide (TLCO) and forced vital capacity (FVC) are the two tests most widely used for this purpose $[2,5]$. As these two PFTs are expressed as percentages of predicted values ( $\%$ predicted), reference equations to calculate predicted values are of critical importance for interpretation.

New reference equations for FVC and for TLCO, based on a large sample of normal subjects, have recently been published by the Global Lung Function Initiative (GLI) [6, 7]. By analysing a large multicentre sample of unselected patients with SSc, we aimed to compare the optimal thresholds of TLCO and of the FVC/TLCO ratio for identification of $\mathrm{PH}$ in these patients, using either previous reference equations $[8,9]$ or the latest GLI equations $[6,7]$.

The population analysed here has been fully described elsewhere [2]. Briefly, PFTs were carried out in SSc patients under stable conditions with standard equipment according to the latest guidelines [10, 11]. All patients underwent Doppler echocardiography at the time of the PFTs and right-heart catheterisation was performed if $\mathrm{PH}$ was suspected. Of the 572 SSc patients who were included, 58 had PH ( 35 had both ILD and $\mathrm{PH}$, while 23 had $\mathrm{PH}$ without ILD) and 514 did not have $\mathrm{PH}$ (313 had no pulmonary involvement, i.e. neither ILD nor PH, while 201 had ILD without PH). The area under the receiver operating characteristic (ROC) curve (the AUC) was used to assess the ability of TLCO and of the FVC/TLCO ratio to discriminate between the presence and the absence of PH. As TLCO is modified not only by PH but also by ILD we performed two analyses, one in SSc patients without ILD $(n=336)$ and another in SSc patients with ILD ( $\mathrm{n}=236)$. The optimal thresholds for both analyses were assessed according to the Youden index of ROC curve analysis in order to maximise both sensitivity and specificity.

By comparison with the previous equations for TLCO [8] and for FVC [9] we observed that, with the GLI equations, mean values of TLCO (\% predicted) were significantly higher (by 4 to $9 \%$, depending on the presence of $\mathrm{PH}$ and/or ILD) and mean values of FVC (\% predicted) were significantly lower (by 9 to $12 \%$ ). Use of the GLI equations also resulted in much lower FVC (\% predicted)/TLCO (\% predicted) ratios than with the previous equations.

In SSc patients without ILD, analysis of the ROC curves regarding the probability of $\mathrm{PH}$ showed that the optimal threshold of TLCO was higher with the GLI equation (70\% predicted versus $60 \%$ predicted) compared with the previous equation (table 1). However, optimal thresholds were similar with both the GLI equation and with the previous equation for SSc patients with ILD. The area under the ROC curve

@ERSpublications

GLI equations provide new cut-offs for TLco for detection of PH in patients with SSc, either with or without ILD http://ow.ly/aCpD30l2zch

Cite this article as: Soumagne T, Guillien A, Chambellan A, et al. Global Lung Initiative equations for pulmonary hypertension screening in systemic sclerosis. Eur Respir J 2018; 52: 1800528 [https://doi.org/ 10.1183/13993003.00528-2018]. 
TABLE 1 Performance of transfer factor of the lung for carbon monoxide (TLCO) and of forced vital capacity (FVC) with different thresholds for detecting the probability of pulmonary hypertension (PH) in patients with systemic sclerosis (SSc), with or without interstitial lung disease (ILD)

\begin{tabular}{|c|c|c|c|c|c|c|c|c|c|c|c|c|}
\hline & \multicolumn{6}{|c|}{ SSc patients without ILD } & \multicolumn{6}{|c|}{ SSc patients with ILD } \\
\hline TLco \% predicted (Crapo) & $0.89(0.84-0.95)$ & $60 \%$ & 78 & 84 & 26 & 98 & $0.76(0.68-0.84)$ & $55 \%$ & 83 & 58 & 26 & 95 \\
\hline \multirow[t]{3}{*}{ TLco z-score $(G L I)^{\#}$} & $0.90(0.85-0.95)$ & -2.02 & 87 & 79 & 23 & 99 & $0.74(0.66-0.82)$ & -3.55 & 77 & 66 & 28 & 94 \\
\hline & & -1.96 & 87 & 77 & 22 & 99 & & -1.96 & 91 & 43 & 22 & 97 \\
\hline & & -1.64 & 87 & 72 & 19 & 99 & & -1.64 & 94 & 36 & 20 & 97 \\
\hline $\begin{array}{l}\text { FVC } \% \text { predicted } / \text { TLCo } \% \\
\text { predicted (GLI) }\end{array}$ & $0.87(0.79-0.95)$ & 1.50 & 77 & 87 & 30 & 98 & $0.73(0.65-0.81)$ & 1.45 & 71 & 67 & 27 & 93 \\
\hline
\end{tabular}

AUC: area under the receiver operating characteristic curve; Se: sensitivity; Sp: specificity; PPV: positive predictive value; NPV: negative predictive value; GLI: Global Lung Initiative. ${ }^{*}$ : a $z$-score $=-1.96$ results when $2.5 \%$ of healthy individuals will be below a cut-off identified as the lower limit of normal (LLN); a z-score $=-1.64$ results when $5 \%$ of healthy individuals will be below a cut-off identified as the LLN.

was significantly higher for SSc patients without ILD than for those with ILD. When TLCO was expressed as a $\mathrm{z}$-score, the optimal threshold value was $\sim-2.00$ for SSc patients without ILD and $\sim-3.50$ for SSc patients with ILD.

For the FVC/TLCO ratio (FVC and TLCO both being expressed as \% predicted values), we identified an optimal threshold that was similar for SSc patients without ILD whatever the equations used. However, this threshold was $\sim 15 \%$ lower for SSc patients with ILD with the GLI equations compared with the previous equations (table 1).

As other authors have recently remarked, PFTs are used for diagnosis and monitoring at the patient level, and percentage of predicted value is the most common way to express results, to define normal status and/or to grade disease severity [12]. Consistency in interpretation of PFTs from one laboratory to another depends at least in part on the choice of reference equation used to standardise measurements [12]. The GLI was formed in 2008 with the aim of improving reference equations in order to standardise the interpretation of PFTs worldwide. The GLI reference equations have been endorsed by all major international respiratory societies and adopted as the recommended reference equations by many national respiratory societies [12]. Given the crucial role of PFTs in screening for $\mathrm{PH}$ in SSc, it was important to make a comprehensive evaluation of the diagnostic value of TLCO and FVC/TLCO with these new equations, which are likely to be the most widely used worldwide in the near future.

There are both pragmatic and pathophysiological reasons for using TLCO as a screening test for PH. First, TLCO is a non-invasive test widely available in pulmonary function laboratories where SSc patients are followed. Secondly, TLCO is very strongly correlated with pulmonary capillary blood volume [2]. This is impaired when pulmonary vessels are remodelled, as is the case in SSc-related PH. In contrast, use of the $\mathrm{FVC/TLCO}$ ratio is less pertinent from a pathophysiological point of view. It should be remembered that TLCO is calculated by the product of the transfer coefficient of the lung for carbon monoxide (KCO) and the alveolar volume $(V A)$ [13]. At least in part $V A$ is dependent on vital capacity and thus depends on FVC. Taken together, the FVC/TLCO ratio, which by design inversely correlates with TLCO/VA, can be considered as a surrogate for KCO. The FVC/TLCO ratio has been used instead of KCO mainly in order to take into consideration the fact that, in SSc, TLCO can be decreased not only because of vascular involvement (i.e. decreased pulmonary capillary blood volume in $\mathrm{PH}$ ) but also because of a restrictive pattern (i.e. decreased FVC in ILD). Nevertheless, when we took into consideration the presence of ILD, information that is in many cases available in patients with SSc, we found that the FVC/TLCO ratio did not have a better diagnostic power for PH than TLCO alone. As such, the usefulness of measuring FVC in this context is therefore unclear.

In conclusion, we provide here cut-off values obtained with the latest GLI prediction equations for the screening of PH. For this purpose, we suggest taking into account TLCO only (and not the FVC/TLCO ratio) with a cut-off of $70 \%$ predicted (or a $z$-score of -2.00) in SSc subjects without ILD and of $50 \%$ predicted (or a z-score of -3.50) in SSc patients with ILD. 
Thibaud Soumagne ${ }^{1,2}$, Alicia Guillien ${ }^{3}$, Arnaud Chambellan ${ }^{4}$, Thông Hua-Huy ${ }^{5}$, Anh Tuan Dinh-Xuan ${ }^{5}$ and Bruno Degano ${ }^{2,3}$

${ }^{1}$ Service de Pneumologie, CHRU de Besançon, Besançon, France. ${ }^{2}$ Service d’Explorations Fonctionnelles Respiratoires, CHRU de Besançon, Besançon, France. ${ }^{3}$ EA 3920, Université de Franche-Comté, Besançon, France. ${ }^{4}$ Institut du Thorax, CHU de Nantes, Nantes, France. ${ }^{5}$ Service de Physiologie Respiratoire, Hôpital Cochin, Université Paris Descartes, Paris, France.

Correspondence: Bruno Degano, Physiologie-Explorations Fonctionnelles, CHU Jean Minjoz, 25030 Besançon Cedex, France. E-mail: bruno.degano@univ-fcomte.fr

Received: March 162018 | Accepted after revision: July 152018

Conflict of interest: None declared.

\section{References}

1 Steen VD, Medsger TA. Changes in causes of death in systemic sclerosis, 1972-2002. Ann Rheum Dis 2007; 66: 940-944.

2 Degano B, Soumagne T, Delaye T, et al. Combined measurement of carbon monoxide and nitric oxide lung transfer does not improve the identification of pulmonary hypertension in systemic sclerosis. Eur Respir J 2017; 50: 1701008 .

3 Humbert M, Yaici A, de Groote $\mathrm{P}$, et al. Screening for pulmonary arterial hypertension in patients with systemic sclerosis: clinical characteristics at diagnosis and long-term survival. Arthritis Rheum 2011; 63: 3522-3530.

4 Schreiber BE, Valerio CJ, Keir GJ, et al. Improving the detection of pulmonary hypertension in systemic sclerosis using pulmonary function tests. Arthritis Rheum 2011; 63: 3531-3539.

5 Coghlan JG, Denton CP, Grunig E, et al. Evidence-based detection of pulmonary arterial hypertension in systemic sclerosis: the DETECT study. Ann Rheum Dis 2014; 73: 1340-1349.

6 Stanojevic S, Graham BL, Cooper BG, et al. Official ERS technical standards: Global Lung Function Initiative reference values for the carbon monoxide transfer factor for Caucasians. Eur Respir J 2017; 50: 1700010.

7 Quanjer PH, Stanojevic S, Cole TJ, et al. Multi-ethnic reference values for spirometry for the 3-95-yr age range: the global lung function 2012 equations. Eur Respir J 2012; 40: 1324-1343.

8 Crapo RO, Morris AH. Standardized single breath normal values for carbon monoxide diffusing capacity. Am Rev Respir Dis 1981; 123: 185-189.

9 Quanjer PH, Tammeling GJ, Cotes JE, et al. Lung volumes and forced ventilatory flows. Eur Respir J 1993; 6: Suppl. 16, 5-40.

10 Miller MR, Hankinson J, Brusasco V, et al. Standardisation of spirometry. Eur Respir J 2005; 26: 319-338.

11 Macintyre N, Crapo RO, Viegi G, et al. Standardisation of the single-breath determination of carbon monoxide uptake in the lung. Eur Respir J 2005; 26: 720-735.

12 Stanojevic S. Standardisation of lung function test interpretation: Global Lung Function Initiative. Lancet Respir Med 2018; 6: 10-12.

13 Hughes JM, Pride NB. Examination of the carbon monoxide diffusing capacity (DL(CO)) in relation to its KCO and VA components. Am J Respir Crit Care Med 2012; 186: 132-139. 